\title{
Advanced Experimental Data Processing for the Identification of Thermal and Strain-Rate Sensitivity of a Nuclear Steel
}

\author{
M. Peroni ${ }^{1}$ (D) . G. Solomos ${ }^{1}$
}

Received: 7 April 2019 / Accepted: 19 June 2019 / Published online: 25 June 2019

(c) The Author(s) 2019

\begin{abstract}
The knowledge of material mechanical behaviour in different physical conditions is necessary to accurately simulate structural response using finite element methods, especially when complex physical processes, such as strain-hardening, large strains etc., are involved. In this context, the material characterization at different temperatures and strain-rates is indispensable, but it is equally essential to properly transform the test data into efficient constitutive equations capable to accurately reproduce the material response. As an alternative to the conventional analytical approach of the stress-strain curve fitting, this investigation examines the adoption of an inverse method that exploits a FEM model to accurately keep account of the specimen stress, strain, and temperature fields. The material parameters of the selected constitutive model are then obtained by using an optimization algorithm that iteratively changes the parameter values to minimize a target function. The algorithm has been implemented in MATLAB using the LS-DYNA FEM solver. In the paper, this method has been applied to the experimental data produced in a test campaign (EU project LISSAC) for a ferritic steel normally employed in nuclear pressure vessels. These data refer to tensile testing under several strain-rate and temperature conditions and include both smooth and notched cylindrical specimens. The constitutive models of Johnson-Cook and Zerilli-Armstrong have been considered for the demonstration of the methodology. The efficiency of the approach in determining the model parameters is critically assessed.
\end{abstract}

Keywords Thermal and strain-rate sensitivities $\cdot$ Inverse methods $\cdot$ Constitutive equation $\cdot$ Parameter identification $\cdot$ Strainrate $\cdot$ Modified Hopkinson bar $\cdot$ SHPB

\section{Introduction}

The knowledge of material mechanical behaviour in different physical conditions is necessary to accurately simulate structural response using finite element methods (FEM). Material properties are rather well known in the elastic regime but several difficulties appear when complex physical processes are involved, such as strain-hardening, large strains, strainrate and thermal sensitivity, damage, etc. This is the case of dynamic problems like impacts or explosions, where components are loaded beyond the elastic regime in a wide range of velocities and temperatures and/or dynamic phenomena cause the material adiabatic heating due to the conversion of plastic work into heat.

M. Peroni

marco.peroni@ec.europa.eu

1 European Commission, Joint Research Centre, Via E. Fermi 2749, 21027 Ispra, VA, Italy
In this context, the material characterization at different temperatures and strain-rates in the laboratory is indispensable, but it is equally essential to properly transform these data into efficient constitutive equations capable to accurately reproduce the material behaviour using FEM codes. In fact, traditional analytical methods, normally adopted to convert experimental data (for example force-displacement curves) in a constitutive material model (a set of stress-strain curves at different temperatures and strain-rates), are overly approximated to obtain accurate simulations. This is mainly due to the fact that in dynamic tests the stress, strain and thermal fields are not uniform (for example necking phenomena in tensile tests) and barely representable with analytical models.

In addition, material models normally adopted in dynamic simulations (Johnson-Cook (J-C) model [1], Zerilli-Armstrong (Z-A) model [2], etc.) may not be able to accurately reproduce the complex mechanical behaviour of all materials used in structural applications. 
In this framework, this paper presents the data elaborations of a past experimental test campaign performed on the ferritic steel 22NiMoCr37 [3-5] normally used in nuclear pressure vessels. The work relates to the completed EU project LISSAC (Limit Strains for Severe Accident Conditions [6]), in which the Joint Research Centre was a partner. The experimental data available from this test campaign refer to tensile testing under several strain-rate and temperature conditions. As previously discussed, the produced stress-strain curves are barely amenable to standard curve fitting techniques, probably due to the geometry of the tested specimen (which causes early necking) and to the specific material behaviour which is not simply described by a conventional material model (especially with respect to the thermal sensitivity).

As an alternative to the conventional analytical approach, this paper examines the adoption of an inverse method to overcome some of the limitations just mentioned. The inverse method investigated [7-19] exploits a FEM model to accurately keep count of the stress, strain, strain-rate and temperature fields in the specimen. The material parameters of the selected constitutive model are obtained by using an optimization algorithm that iteratively changes the parameter values in order to minimize a target function. The target function is chosen to be simply the mean absolute relative error (MAPE) between the experimental force-displacement curve of each test elaborated and the corresponding simulated one. The algorithm has been implemented in MATLAB using the LS-DYNA FEM solver, where an automatized procedure has been developed that requires as input only a FEM model of the specimen and the force-displacement curve of each test considered.

The expected main advantages of the proposed procedure could be summarized as follows [20,21]:

(a) increase of data interpolation accuracy due to the meticulous reproduction of the stress, strain and temperature fields in the specimen without unnecessary simplifications, such as the Bridgman correction;

(b) automation of data elaboration independent of specimen geometry or test boundary conditions;

(c) inclusion of other test or post-mortem measurements in the optimization target function, like for example the specimen profile or the final diameter of the necking section.

Sections "Specimens and Experimental Apparatuses" and "Test Results" of the paper present an overview of the experimental test campaign performed in the LISSAC project and the main data acquired. These data consist of three sets, which come from a series of tensile tests of the ferritic steel $22 \mathrm{NiMoCr} 37$ conducted at different temperatures $\left(20{ }^{\circ} \mathrm{C}, 400{ }^{\circ} \mathrm{C}, 850{ }^{\circ} \mathrm{C}\right.$ ) and two strain-rate levels (about
$0.002 / \mathrm{s}$ and $250 / \mathrm{s})$. The first set refers to a cylindrical specimen geometry (gage section of $3 \mathrm{~mm}$ diameter and of $5 \mathrm{~mm}$ length) used to study the thermal and strain-rate influence on the strain-hardening material behaviour. The second set refers to a notched specimen geometry used to study the effect of triaxiality on the material behaviour. The specimens have the same dimensions as above but with a semicircular notch in the middle. The third set of data refers to tests performed on larger notched specimens with geometry similar to the previous one but scaled-up seven times. For these large specimens only dynamic tests (at about 200/s) were conducted using the HopLab facility (the very large Hopkinson bar at the JRC-Ispra).

"Analytical Test Data Elaboration" section of the paper describes a conventional analytical elaboration of the experimental data emphasizing the limitations of this approach especially when the material behaviour at large strain levels and high temperatures is required. The material parameters obtained here for the constitutive laws considered ( $\mathrm{J}-\mathrm{C}$ and $\mathrm{Z}-\mathrm{A}$ models) are the starting point of the inverse method described next.

"Inverse Method Elaboration" section presents in detail the implementation, the advantages and the drawbacks of the proposed inverse method for the identification of the material model parameters. In this context, the block diagram of the developed algorithm is analysed and thoroughly discussed. Considerations about the material models available in literature are discussed aiming to overcome some limitations observed in the interpolation of experimental data. Finally, using in FEM calculations the optimized material parameters previously extracted, verifications are conducted through comparisons between experimental data from notched specimens (data-sets two and three) and simulated ones in order to assess the performance of the proposed algorithms.

\section{Specimens and Experimental Apparatuses}

The steel for the specimens of the LISSAC project has been furnished by the LISSAC partner MPA (Materialprüfungsanstalt, Stuttgart). The experimental pressure vessel BIBLIS-C existing there, has been dismantled and cut to pieces, according to a predefined cutting plan. Figure 1 shows the part of the cylindrical section of the original vessel used for the construction of the specimens. The several segments produced are shown schematically in the same figure. These segments or smaller pieces thereof have been distributed to the LISSAC partners according to the respective needs in material. The steel of the vessel is the ferritic steel 22NiMoCr37, a material that is quite similar to the more known A $508 \mathrm{Cl} 2$.

According to the cutting plan, three different specimen geometries have been obtained, as reported in Fig. 2. As a 


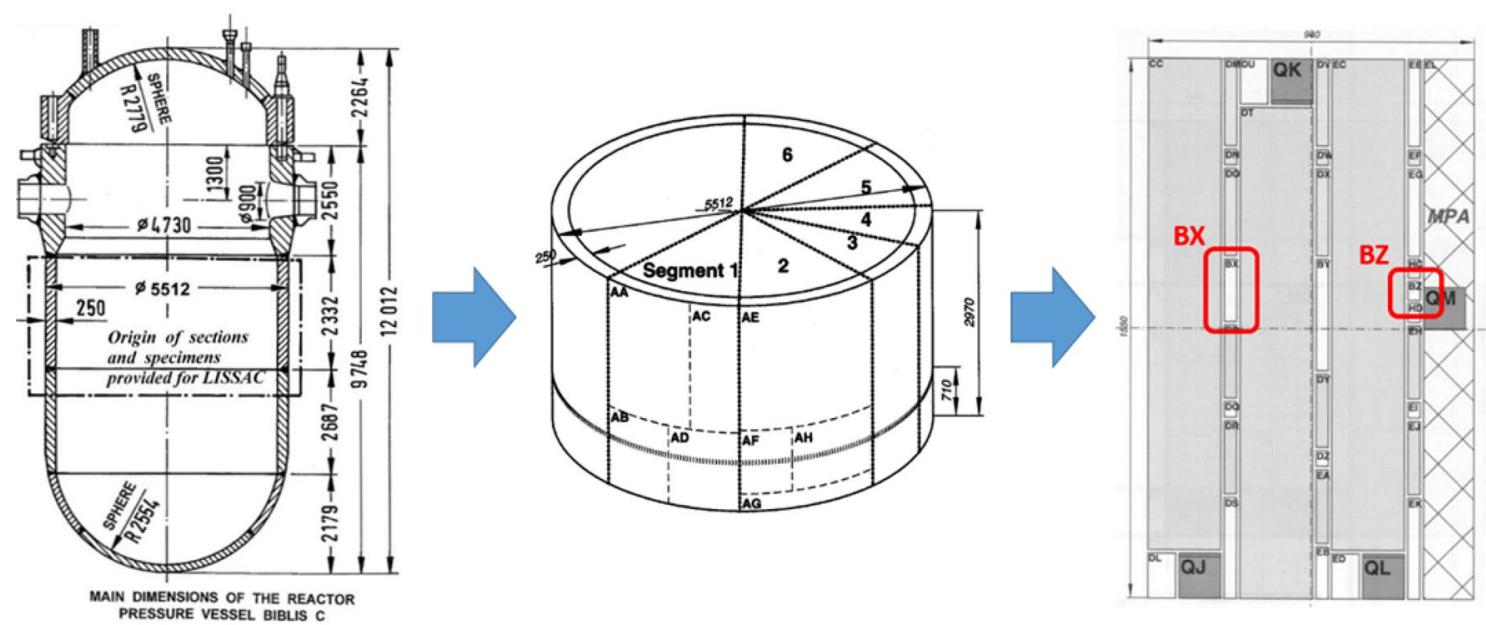

Fig. 1 Cutting plan for LISSAC specimens
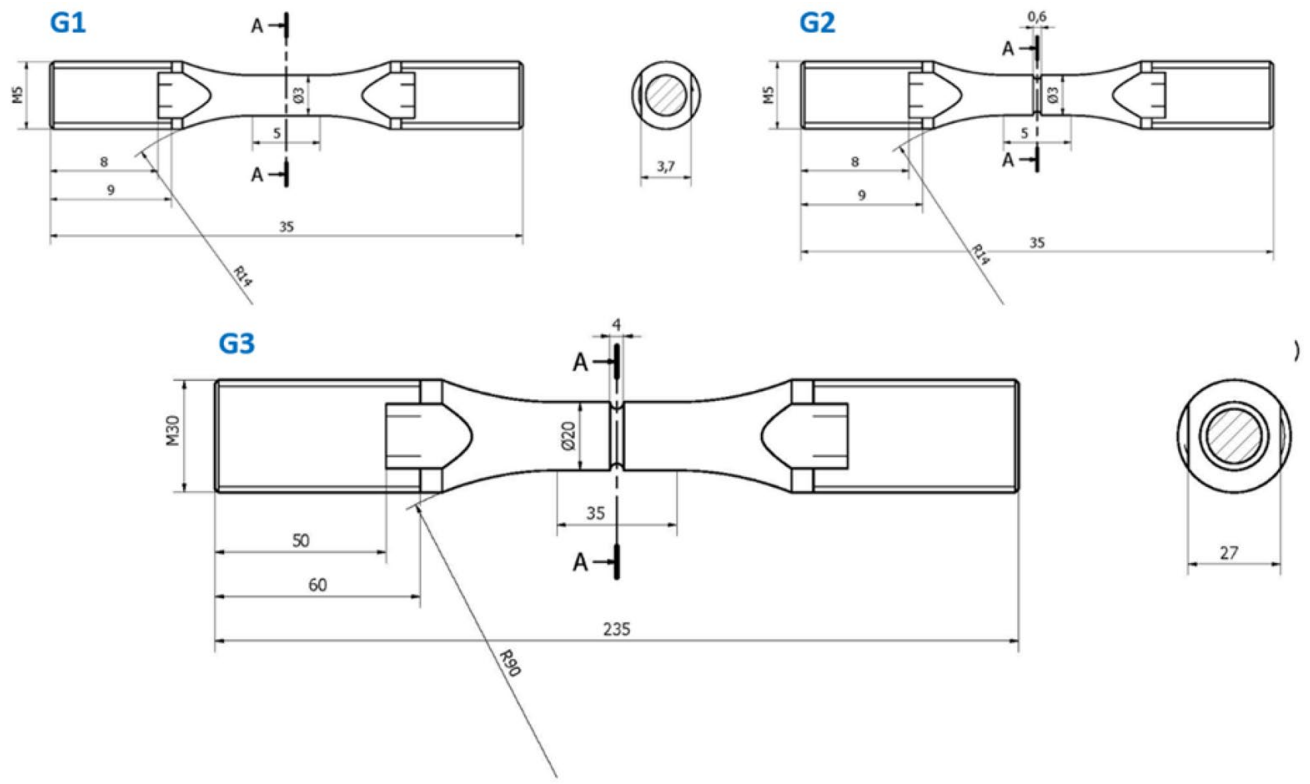

Fig. 2 Specimen geometries and dimensions (in $\mathrm{mm}$ )

general rule, all specimens have been cut along the longitudinal axis direction of the original vessel from material positioned at $1 / 4$ thickness from the shell's outside surface. The first geometry adopted for the material mechanical characterization is the classical tensile threaded-ends geometry, typically used in dynamic characterization at the JRC (geometry G1). These specimens have been cut from the upper part of segment 4 , from the positions BX and BZ.

A second batch of specimens has been cut from the same vessel section with a geometry G2 very similar to the specimens for the material characterization. The only difference is represented by a semi-circular notch in the middle of the gage length, as shown in Fig. 2, that modifies the stress field in the specimen. The third batch of specimens has the same geometry as the previous notched specimen but with a larger scale (G3) and it has been designed for investigating possible size-effects.

The above choice of the quarter-thickness position has been applied for reducing possible positional effects in the properties of the material. However, an extensive material quality assurance study, conducted by the LISSAC partner Framatome, has shown among others that practically no differences exist in the mechanical properties of this vessel steel along the longitudinal and the circumferential vessel directions. Further, in order to minimize fabrication differences, the construction of the specimens of the 
JRC has been ordered together with those for the partners PSI and EMPA (Paul Scherrer Institut and Eidgenössische Materialprüfungs und Forschungsanstalt, $\mathrm{CH}$ ) to a single machine shop.

Table 1 summarizes the experimental plan and the number of test replicates carried out at the Joint Research Centre (JRC). Testing for the range of strain-rates investigated and the different specimen geometries has been performed by means of three different apparatuses: a screw tensometer for the static tests and two modified Hopkinson bars for the dynamic ones [22,23]. Since tensometers and conventional Hopkinson bars are regularly used in the material characterization, only a brief description is provided below for the Hopkinson bar used for the large size specimens (geometry G3). The diagram of this large Hopkinson bar (HopLab) developed at the JRC is shown in Fig. 3.

The HopLab, with its unique features, is one of the largest existing Hopkinson bar apparatuses with a length of more than $200 \mathrm{~m}$ and a bar diameter of $72 \mathrm{~mm}$ made of high strength steel (MSK). Different from a classical split Hopkinson bar, where a projectile is used to generate the loading pulse, a high strength steel cable, which is the physical continuation of the input bar, is used. Through statically pre-tensioning and suddenly releasing this cable, rectangular force pulses of up to $1 \mathrm{MN}, 250 \mu \mathrm{s}$ rise-time and $40 \mathrm{~ms}$ duration can be generated and applied to the specimen tested.

The high temperature tests have been conducted by using in-house made ovens with resistance heating. No inert atmosphere has been used, and the effect of possible oxidation (with subsequent reduction of the cross-sectional area) has not been evaluated. It is, however, judged that this effect cannot be significant since the duration of the heating phase and of the test itself is rather short. However, special care has been taken of cooling down the Hopkinson bar ends in order to avoid any alterations in the wave propagation parameters. For this purpose bespoke hollow rings with cold water circulating through them have been fitted at the bar ends close to the specimen.

\section{Test Results}

An overview is provided below of the experimental data obtained in the project at the JRC and employed in the current study. All data curves presented in this section are displayed in terms of force and displacement, either directly recorded or analytically derived in the Hopkinson bar tests, because it is in this form that they are used in the inverse

Table 1 Experimental work matrix

\begin{tabular}{llllll}
\hline Test type & $\begin{array}{l}\text { Specimen } \\
\text { geometry }\end{array}$ & $\mathrm{T}=20^{\circ} \mathrm{C}$ & $\mathrm{T}=400{ }^{\circ} \mathrm{C}$ & $\mathrm{T}=600{ }^{\circ} \mathrm{C}$ & $\mathrm{T}=850{ }^{\circ} \mathrm{C}$ \\
\hline Static test $(0.001 / \mathrm{s})$ & $\mathrm{G} 1$ & 3 & 3 & 2 & 3 \\
Dynamic test (250/s) & $\mathrm{G} 1$ & 4 & 3 & - & 4 \\
Static test (0.001/s) & $\mathrm{G} 2$ & 4 & 4 & - & 3 \\
Dynamic test (250/s) & $\mathrm{G} 2$ & 4 & 4 & - & 3 \\
Dynamic test (200/s) & $\mathrm{G} 3$ & 2 & 2 & - & 2 \\
\hline
\end{tabular}
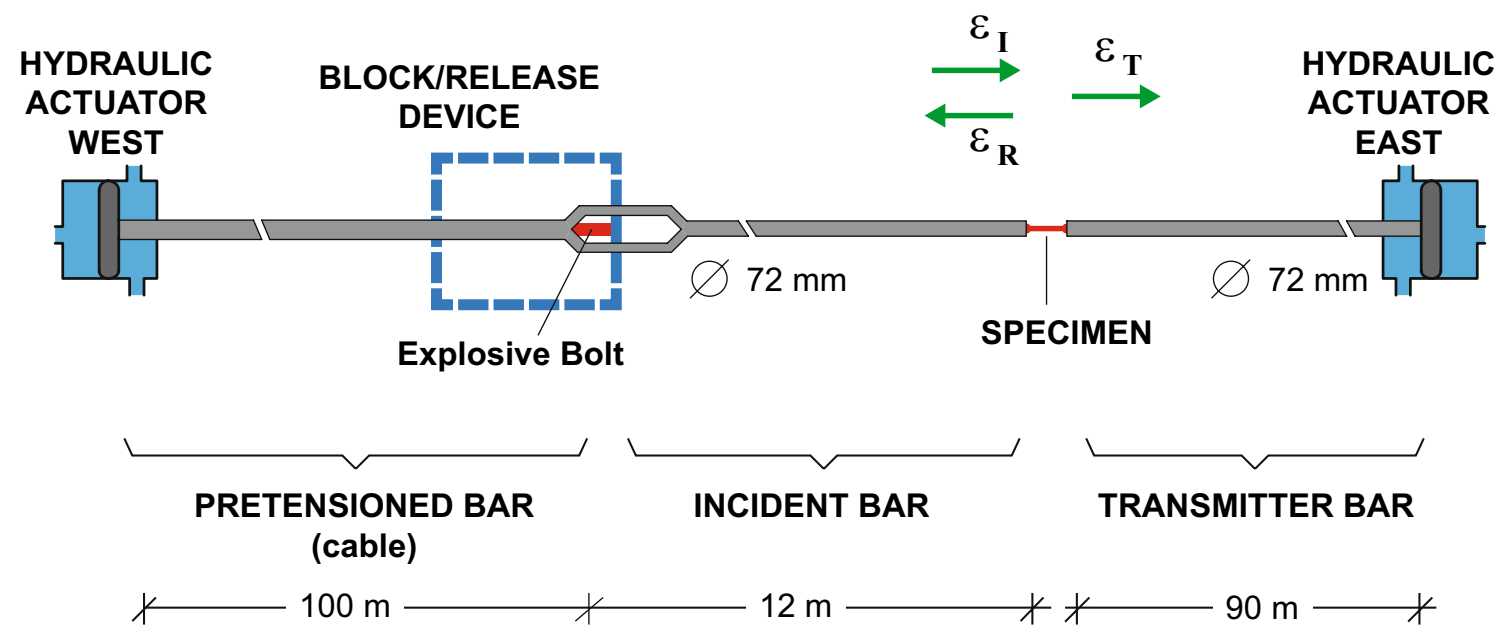

Fig. 3 HopLab facility: large Hopkinson bar for dynamic mechanical testing 
method investigation in "Inverse Method Elaboration" section. However, typical analytical elaborations (based on stress-strain diagrams) are also carried out in "Analytical Test Data Elaboration" section.

Figure 4 shows experimental data obtained from testing of smooth cylindrical specimens (G1), statically and dynamically at different temperatures. The instability at yielding is manifested in the dynamic testing for all three temperatures. A non-negligible data scattering is also observed, considering the high homogeneity of the tested material, and this strength variability could only be justified by the fact that the specimens have been cut directly from a real component, which has undergone many and diverse loading cycles. For the modelling purposes two main observations can be made from the experimental data reported in Fig. 4: (a) The steel $22 \mathrm{NiMoCr} 37$ exhibits a notable strain-rate and thermal sensitivity in the test conditions analysed; (b) the thermal sensitivity is strongly strain-rate dependant and for this reason it will be hardly representable with simplified mechanical models based on the independency of strain-rate and thermal sensitivity, like for example the $\mathrm{J}-\mathrm{C}$ model.

An additional consideration can be drawn about the oscillations in the curves related to static tests at $850{ }^{\circ} \mathrm{C}$ and to a lesser extent at $400{ }^{\circ} \mathrm{C}$. These oscillations are probably not related to the material behaviour but rather to an imperfect velocity control of the tensometer adopted. Anyway, these perturbations do not dramatically affect the interpretation of experimental data.

Figure 5 shows the experimental data obtained from the second batch of specimens with the notched geometry (G2), tested under the same conditions.

The general material behaviour, consisting of a noticeable strain-rate and thermal sensitivity, seems to be confirmed by the notched-specimen tests, too. Also in this case strain-rate sensitivity appears to be thermal dependent. Obviously, due to the increased triaxiality and the stress concentration phenomena induced by the notch, the maximum displacements are substantially reduced. In addition, no more oscillations

a
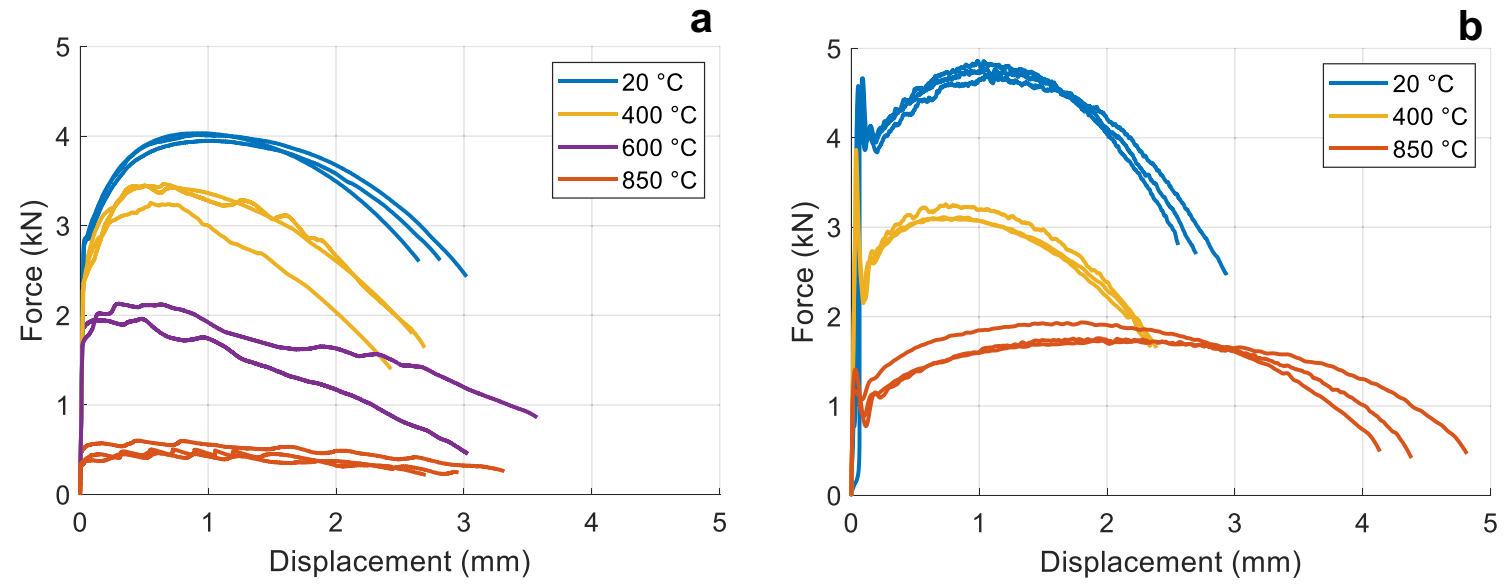

Fig. 4 a Static and $\mathbf{b}$ dynamic test curves from smooth cylindrical specimens (geometry G1)
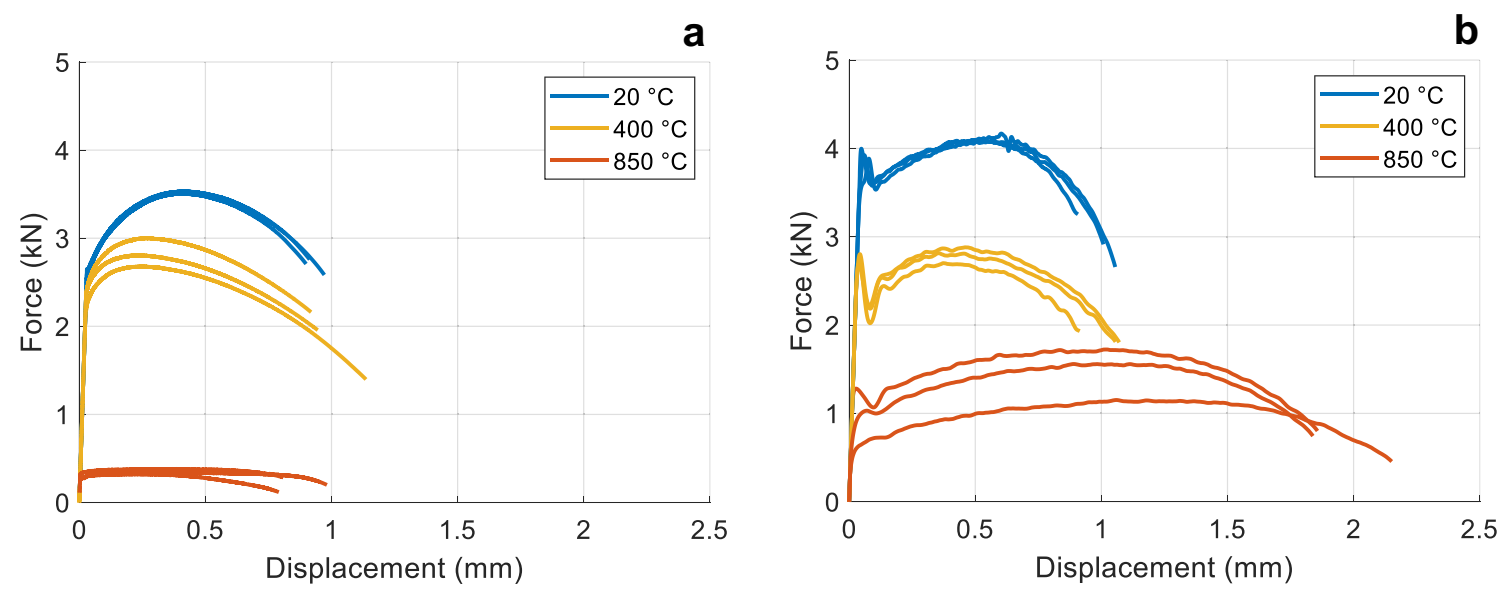

Fig. 5 a Static and b dynamic test curves from small notched cylindrical specimens (geometry G2) 
are present in the force-displacement curves of the high temperature tests.

The same behaviour can be observed in Fig. 6, which summarises experimental dynamic tests performed with the large notched specimens. Dynamic test curves of Figs. 5b, 6 appear to have the same morphological shape save for possible size-effects. The stronger oscillations in the curves of Fig. 6 are probably induced by the additional masses of the specimen fixtures used at the HopLab, which anyway do not prevent a correct data interpretation. Finally, a reduced experimental scattering can be observed in this last test batch, most likely due the greater and therefore more representative material volume of the tested specimens.

A final, important consideration concerns the tests performed at $850{ }^{\circ} \mathrm{C}$. As reported in the ASTM standard for this material class [3-5], at this temperature the material is close or even beyond the austenizing temperature (for example for AS 508 grade 4 this is about $840{ }^{\circ} \mathrm{C}$ ). In this context, since the specimens were maintained at $850{ }^{\circ} \mathrm{C}$ for a relatively long time (about 5 min for small specimens), phase transformations could have occurred during the heating phase. For this reason, the validity of the test data performed at this

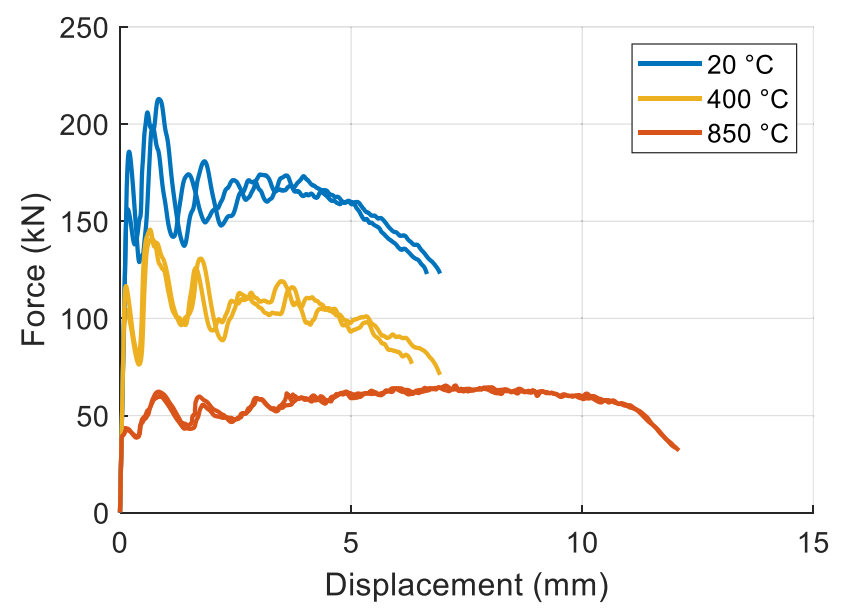

Fig. 6 Dynamic test curves from large notched cylindrical specimens (geometry G3) temperature is questionable for the purposes of the parameter identification of the material model. They are considered as outliers during the analysis due to the fact that in this case the specimens could have a different material structure compared to those at lower temperatures.

\section{Analytical Test Data Elaboration}

When a standard mechanical test is performed on metals, the measured quantities usually concern global properties of the specimen analysed. Standards macroscopic quantities are the forces and the displacements measured at the specimen ends (or in a portion of the gage length). These signals must be next elaborated to obtain the true stress-true plastic strain curve, which is the classical input of commercial FEM codes.

With reference to a standard tensile or compression test, the diagram in Fig. 7 shows the usual elaboration procedure of the experimental data. Starting from the force-displacement curve measured by the testing machine, the engineering stress-strain curve is obtained assuming uniaxial stress uniformity in the specimen (or at least in the uniform gage length for tensile specimens). With the hypothesis of volume conservation in the plastic regime and subtracting the elastic strain component, it is possible next to obtain the material true stress-true plastic strain curve that can be fitted with a material model, such as J-C, etc. In addition, for tensile tests, only the experimental curve before the maximum stress can be directly used to identify the material model parameters because after this point the uniaxial hypothesis is no more verified. Certainly, the adoption of more sophisticated measurement systems (extensometers and Digital Image Correlation systems) or elaboration techniques (for example the Bridgman correction for compensating for triaxiality in the post necking zone in tensile tests or other compensation algorithms) could overcome some of these problems, however at the expense of dramatically increasing the test or data elaboration complexity.

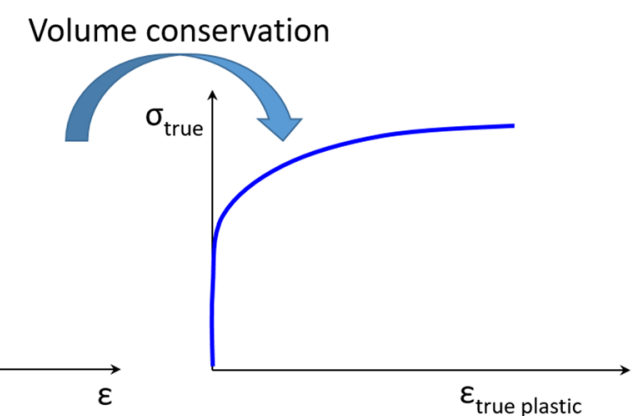

Fig. 7 Diagram of analytical elaboration procedure 
Accordingly, the standard elaboration results of the experimental data concerning the cylindrical specimen (geometry G1) are presented below using only the pre-necking phase of each test and transforming the force-displacement curves following the above-described steps. These data constitute the starting point for the optimization procedure undertaken next. It is finally mentioned that the temperature increase during the dynamic tests (heating due to adiabatic conditions) has not been calculated because it is negligible for the small range of strain considered in this analysis.

Figs. 8, 9 present the two data series (static and dynamic) in terms of true stress-true plastic strain of the $22 \mathrm{NiMoCr} 37$ steel at the tested temperatures. The experimental data have been fitted with two well-known material models implemented in the majority of FEM commercial codes, the J-C model and the Z-A model models, both of which are able to describe a strain-hardening behaviour and strain-rate and temperature dependence. In both cases, an optimization procedure, implemented in Matlab, has been used to identify the model parameters that produce a best fit to the experimental curves. As explained in the previous paragraph, data concerning tests at $850{ }^{\circ} \mathrm{C}$ are not used in this optimization run but they are in any case shown in the diagrams as an extrapolation exercise.

The classical J-C model with the multiplicative formulation is adopted in the form:

$$
\begin{gathered}
\sigma=\left(A+B \times \varepsilon_{p}^{n}\right) \times\left(1+c \times \ln \dot{\varepsilon}^{*}\right) \times\left(1-T^{* m}\right) \\
\text { with } \dot{\varepsilon}^{*}=\frac{\dot{\varepsilon}_{p}}{\dot{\varepsilon}_{0}} \text { and } T^{*}=\frac{T-T_{r}}{T_{m}-T_{r}}
\end{gathered}
$$

where, $\sigma$ is the Von Mises flow stress, $\varepsilon_{p}$ the equivalent plastic strain, $\dot{\varepsilon}^{*}$ the dimensionless plastic strain-rate, $\dot{\varepsilon}_{p}=$ test plastic strain-rate, $T^{*}$ the homologous temperature, $T$ the specimen absolute temperature and $A, B, n, c$ and $m$ are the five material constants. According to the above analysis, the model parameters that best fit the experimental data (using $\dot{\varepsilon}_{0}=1 / \mathrm{s}$, melting temperature $T_{m}=1400^{\circ} \mathrm{C}$ and room temperature $T_{r}=20^{\circ} \mathrm{C}$ ) are reported in Table 2 .
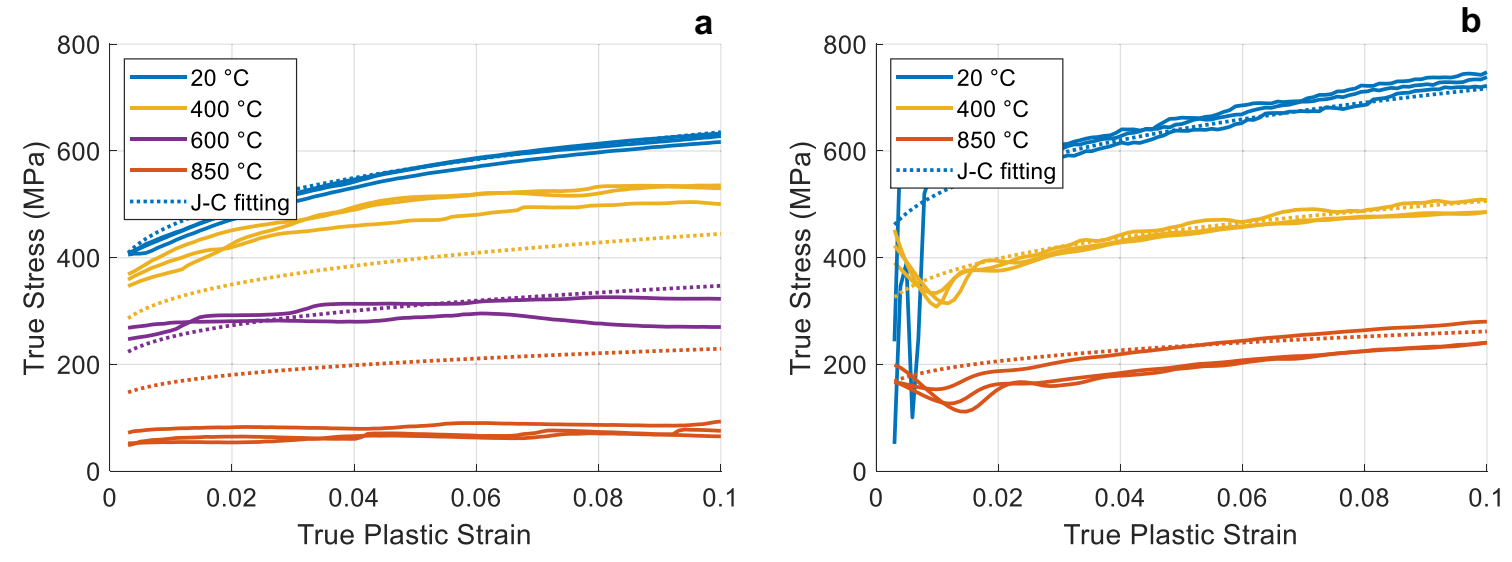

Fig. 8 a Static and $\mathbf{b}$ dynamic true stress-true plastic strain curves and J-C fitting

a

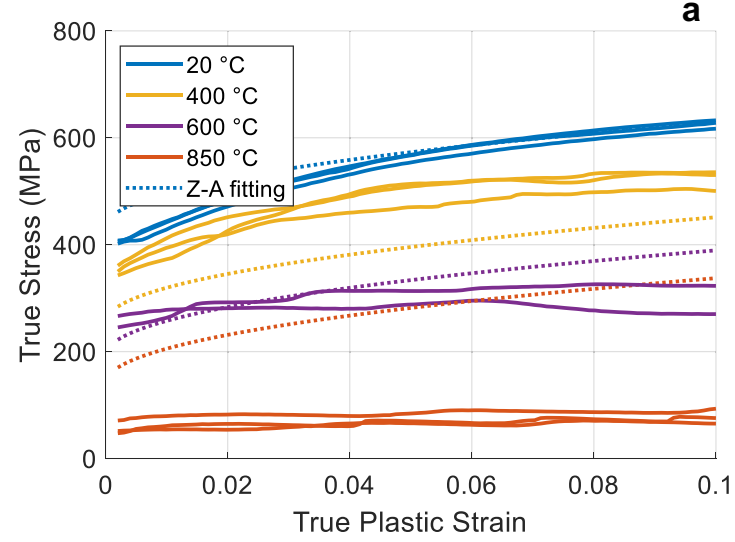

b

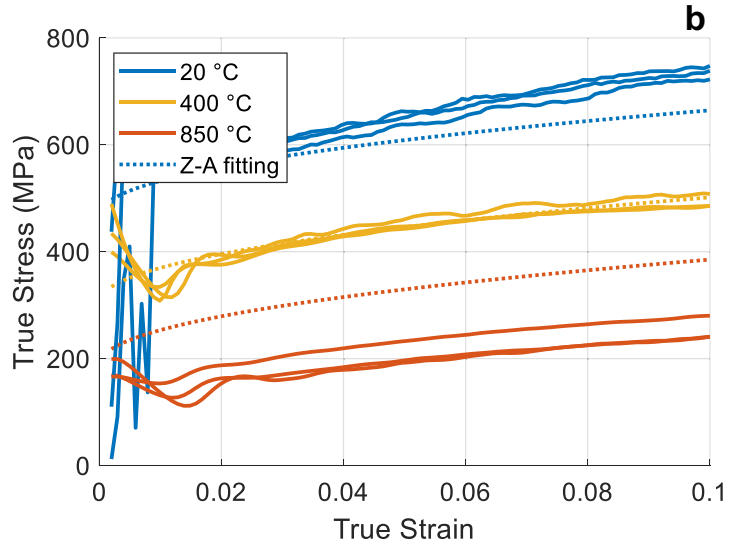

Fig. 9 a Static and b dynamic true stress-true plastic strain curves and Z-A fitting 
Table 2 Model parameter values according to the analytical fitting approach

\begin{tabular}{lllllll}
\hline Johnson-Cook model & $A(\mathrm{MPa})$ & $B(\mathrm{MPa})$ & $n$ & $c$ & $m$ & \\
& 303.0 & 703.9 & 0.325 & 0.024 & 1.107 & \\
Zerilli-Armstrong model & $c_{0}(\mathrm{MPa})$ & $K(\mathrm{MPa})$ & $n$ & $B_{0}(\mathrm{MPa})$ & $\beta_{0}$ & $\beta_{1}$ \\
& 285.7 & 593.5 & 0.477 & 629.8 & 0.00136 & 0.000024 \\
\hline
\end{tabular}

As seen in Fig. 8, the $\mathrm{J}-\mathrm{C}$ model (dashed lines) is not able to satisfactorily interpolate the experimental data, even without referring to the tests at $850{ }^{\circ} \mathrm{C}$. For implementing the optimization procedure and making quantifiable comparisons the mean absolute percentage error (MAPE) is used, defined as:

$M A P E=\frac{100}{n} \sum_{t=1}^{n}\left|\frac{A_{t}-F_{t}}{A_{t}}\right| \%$

where $A_{t}$ is the actual value (in this case the experimental true stress-true plastic strain curve), $F_{t}$ is the forecast value (the $\mathrm{J}-\mathrm{C}$ fitting) and $n$ is the length of the vectors $A$ or $F$.

Specifically, a $M A P E$ of $8.03 \%$ is emblematic of a poor fitting model. This is clearly due to the fact that the strainrate sensitivity is thermal dependent which is in contrast with the hypothesis of the $\mathrm{J}-\mathrm{C}$ model. On the other hand, the $\mathrm{J}-\mathrm{C}$ model fits rather better the dynamic data than the static ones, also considering the tests performed at $850{ }^{\circ} \mathrm{C}$.

In alternative to the $\mathrm{J}-\mathrm{C}$ model, the $\mathrm{Z}-\mathrm{A}$ model for $\mathrm{BCC}$ materials has been employed in the following additive formulation:

$\sigma=c_{0}+K \times \varepsilon_{p}^{n}+B_{0} \times \exp \left[-\left(\beta_{0}-\beta_{1} \times \ln \dot{\varepsilon}_{p}\right) \times T\right]$

where, the symbols have the same meaning as above, and $c_{0}, K, n, B_{0}, \beta_{0}$ and $\beta_{1}$ are the six material constants. For this case, the model parameters that best fit the available experimental data are also reported in Table 2. Figure 9 shows the fitting of the experimental data using the $\mathrm{Z}-\mathrm{A}$ model derived.

A value of $M A P E=13.30 \%$ is attained with the $\mathrm{Z}-\mathrm{A}$ model, which indicates that the experimental data are no better interpolated compared to the $\mathrm{J}-\mathrm{C}$ model. As noted above for the $\mathrm{J}-\mathrm{C}$ model, also the $\mathrm{Z}-\mathrm{A}$ model fits more accurately the dynamic data than the static ones. Differently from the J-C model, the Z-A model's capability in terms of extrapolation is not satisfactory, as shown by the predicted material behaviour at $850^{\circ} \mathrm{C}$.

As a general comment for this approach, it can be concluded that both $\mathrm{J}-\mathrm{C}$ and $\mathrm{Z}-\mathrm{A}$ models are not capable to correctly fit the complex behaviour of the tested material, which seems not to be representable by a purely multiplicative $(\mathrm{J}-\mathrm{C})$ or a purely additive $(\mathrm{Z}-\mathrm{A})$ model. In fact, observing Fig. 9a it appears that the static material behaviour could be fitted efficiently with a multiplicative model, as the different curves seem to be scaled by a thermally dependent factor. On the contrary, dynamic data (Fig. 9b) appear to be simply translated, in which case an additive model would be more suitable for the interpolation. This mixed additive/ multiplicative behaviour could probably be efficiently fitted with advanced complex models (in terms of formulation and number of parameters), which, however, are not commonly implemented in commercial FEM codes.

For example, in the Klepacko-Rusinek K-R model [24] the von Mises flow stress is obtained by the sum of two components: the internal stress (linked to the material strainhardening characteristics) and the effective stress (the components due to the so-called thermal activation that is only a function of strain-rate and temperature).

\section{Inverse Method Elaboration}

The elaboration procedure presented above is the standard methodology normally adopted in order to fit experimental data obtained from tensile/compression/shear tests with a specific material model. As previously presented, this procedure is based on hypotheses that are not totally satisfied especially when the specimen geometry is constrained by the test equipment, as is the case in Hopkinson bar dynamic tests. The two main factors that reduce the accuracy of analytical interpolation procedures of such data are:

\section{Rough Approximation of the Specimen Stress- Strain Field}

This is probably the main factor affecting the accuracy of the analytical procedures. As already mentioned, the stress fields generated during a mechanical characterization test are sometimes not homogeneous and hardly representable with analytical models. In particular, in high strain-rate tests, because of the reduced specimen length (requirement of the Hopkinson bar techniques), data are often characterised by early necking and for this reason by a non-uniform uniaxial stress field. As a consequence, there is a strong variability of the strain-rate in the specimen that, once again, makes difficult the accurate evaluation of its influence on the material behaviour. Finally, in a compression test friction at the specimen ends can dramatically affect the stress-strain field, as normally demonstrated by the barrel shape assumed by the specimens. 


\section{Poor Approximation of Specimen Thermal Field}

The estimation of the thermal field of the specimen is a key point for accurate experimental data interpolations especially when thermal sensitivity is involved. Since static tests are practically isothermal, when the deformation velocity increases tests become more and more adiabatic and the specimen temperature tends to rise due to the conversion of plastic work into heat. This phenomenon is strongly strain dependent and for this reason also the thermal field is far from uniform for large strains. In addition, even at intermediate velocities heat transportation phenomena (conduction and convection) can modify the thermal field making it hardly representable analytically.

As found in the literature several researchers have proposed analytical techniques to overcome the just mentioned approximations but this approach normally increases rapidly the complexity of data elaboration or it needs additional and sophisticated measurement systems. The Bridgman correction of post-necking phase in tensile tests is a good example in this context: a sophisticated algorithm can be applied to experimental data that involve additional measurements such as the diameter and the curvature of the necking profile. Also the wider availability of image processing algorithms (e.g. Digital Image Correlation) may overcome some of the approximations of the analytical procedures, allowing a more accurate strain field evaluation, but even this may not be totally satisfying. The strain field evaluation concerns only the surface of the specimen and, in addition, it requires special hardware and software instrumentation that increases substantially the testing costs. Combining several advantages, the application of DIC associated to the virtual field method [25] appears to be a new promising technique in this direction.

However, due to the increasing computational capability of modern PCs, an alternative approach has been investigated in literature, based on a hybrid methodology that involves experimental testing and mechanical modelling using FEM methods. For example, some authors have suggested to identify the post-necking behaviour by comparing the experimentally measured stress-strain curve with FEM simulations [7, 8]. In these papers, the elasto-plastic constitutive parameters are obtained by minimizing the difference between the experimental curves (in general force-displacement curves) and the simulated ones. Other works [9-12] have further proposed to identify the constitutive material model parameters using more complicated tests, where complex loading conditions or geometries are adopted.

Figure 10 shows the general flow-chart of these hybrid methodologies commonly known as inverse methods. The operating principle of the technique is rather simple: a proper material model is chosen and each experimental test is simulated using a FEM code modelling faithfully the same

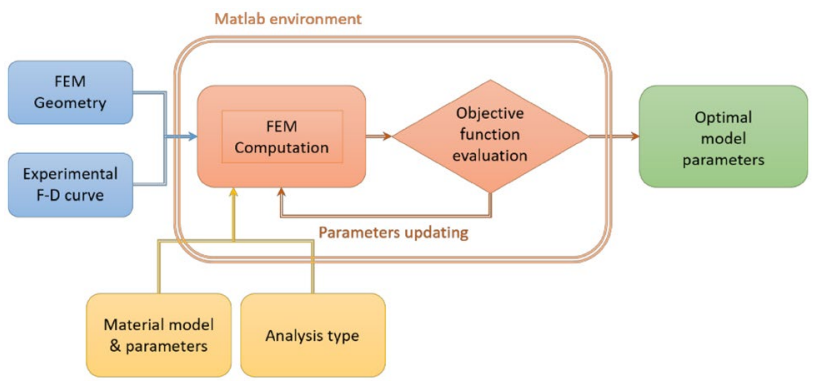

Fig. 10 Diagram of inverse method currently implemented

loading and boundary conditions. At this point, starting from an initial parameter set, an optimization algorithm runs the models iteratively changing the material parameter values. Comparing some experimentally measured quantities with the analogous ones computed by the FEM model it is possible to reach an optimal material parameter set that accurately reproduces the experimental data.

The experimentally measured quantities can be of different type, for example, displacements [13, 14] or velocity fields [15], resonance frequencies [16, 17], applied forces [12], etc. This hybrid approach has the potentiality to overcome many of the limitations of the analytical fitting methodology.

In particular, inverse methods accurately reproduce mechanical and thermal fields generated in the specimen. These fields are modelled with a FEM code without any a priori assumption. In this context, particular specimen geometries (i.e. Hopkinson bar technique) or non-uniformities (i.e. necking) are automatically and effectively considered. Moreover, thermal phenomena (i.e. adiabatic heating or heating conduction) can be easily kept into account. Finally, also non-linear problems, like the friction between the specimen and the platens in a compression test, can be appropriately modelled.

These methods can increase accuracy by adding additional measures. The core of an inversion method is the optimization function that compares experimental quantities and the analogous numerically predicted ones. The simplest choice is to use global quantities such as displacements, forces or velocities that are normally easier to measure during a mechanical test. The optimization function can also incorporate additional global or local measurements (i.e. from strain gages placed at some specimen position, etc.). Moreover, DIC or post mortem data can be readily introduced in the optimization function to increase the interpolation data [9-11, 19]. Finally, if the numerical model is solved with a thermo-mechanical coupled solver, additional thermal measurements can be efficiently incorporated to increase data accuracy or accelerate the algorithm convergence. 
Inverse methods can be automated. An inverse method can be easily structured as an automated procedure applicable to different kinds of tests and materials. In addition, the required modelling competencies are nowadays easier found than the corresponding analytical ones, and if a robust methodology has been implemented, the procedure in principle can be operated by less experienced researchers.

Since, as argued above, inverse methodologies can reduce the experimental effort for an accurate mechanical characterization, their drawbacks mainly concern their computational requirements. In fact, the iterative solution of a FEM model can be highly time consuming if the model dimension is not properly and reasonably designed.

As shown in the diagram in Fig. 10, the optimization procedure has been implemented in the Matlab environment exploiting its great capability in terms of global and local optimization algorithms. In practice, the algorithm loads a geometry and an experiment file that includes all measurements, in this case the curves presented in "Test Results" section and the post mortem measure of the minimum necking diameter. The user can choose the type of analysis (mechanical or thermo-mechanical coupled), the material model, the model geometry (axisymmetric or 3D) and the tests that will be used for the optimization. At this point, a series of FEM models are automatically generated (one for each chosen test) with the same set of material parameters, they are solved using the LS-DYNA software and experimental-computational quantities are compared. Minimizing the optimization function (basically the MAPE between experimental and simulated measures) the optimal parameter set that produces the best fit of the experimental data is obtained.

For what concerns the FEM models adopted in this investigation, all the results presented have been obtained using axisymmetric models (to reduce the computation time) with element size in the specimen gage length of about $0.2 \mathrm{~mm}$. The testing boundary conditions have been formulated in terms of displacements applied to the specimen ends using the displacement time histories recorded experimentally. The force is readily extracted by computing the resultant through a section perpendicular to the specimen longitudinal axis.

To demonstrate the potential of the method Fig. 11a shows the comparison between experimental-simulated data using only one test (quasi-static test at $20{ }^{\circ} \mathrm{C}$ on cylindrical specimen) and employing the J-C model. Considering the force-displacement curves, it is noticed that for the curve obtained using as input the material parameters determined in "Analytical Test Data Elaboration" section (seed parameter set of the optimization) the comparison with the experimental curve is acceptable only in the pre-necking phase but very poor when the whole curve is considered (MAPE $=7.6 \%)$. This is reasonable considering that for the analytical interpolation only the pre-necking data have been used. Passing next to the inverse method and the $\mathrm{J}-\mathrm{C}$ model with three parameters $(A, B, n)$, it is seen that the accuracy of simulated results dramatically increases, reaching a negligible optimization error $(M A P E=0.4 \%)$ with the two curves (experimental-simulated) practically overlapping. Repeating the inverse method with only two J-C model active parameters ( $B$ and $n, A$ not being used) results in an interpolation which remains satisfactory $(M A P E=0.8 \%)$, where, of course, the parameter domain is reduced and the convergence of the inverse method is faster.

The above-determined values of parameters are used next to investigate the predictive capabilities of the $\mathrm{J}-\mathrm{C}$ model. Close agreement between the calculated prediction and the experimental value can be observed for the post mortem measure of the minimum necking diameter, as reported in Fig. $11 \mathrm{~b}$. With reference to this quantity the interpolation error reduces from MAPE $=29.5 \%$ (test simulated with a

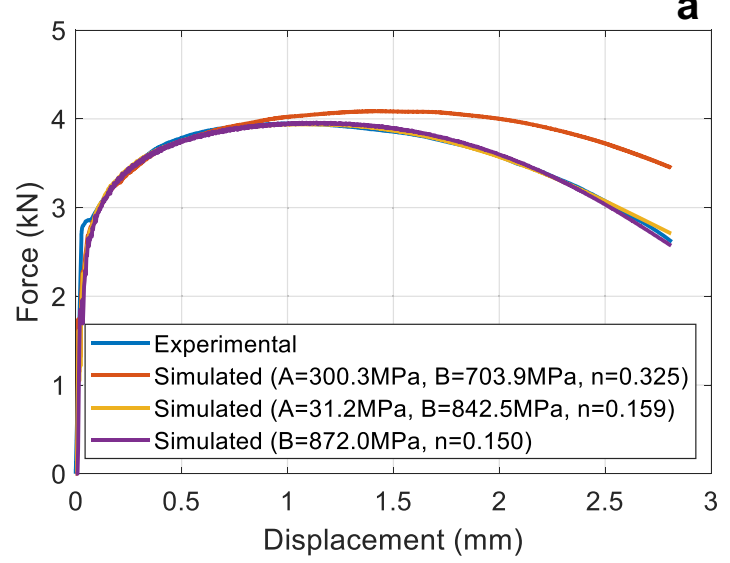

b

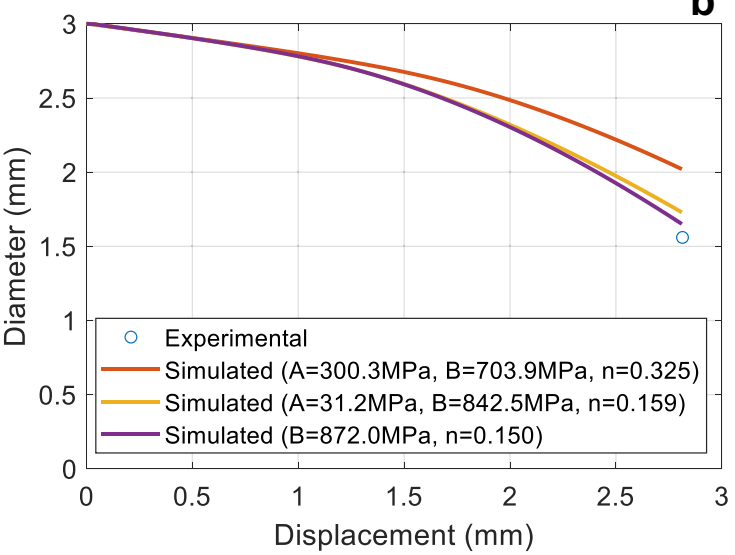

Fig. 11 Comparison experimental-simulated data for quasi static test at $20{ }^{\circ} \mathrm{C}$ (geometry G1) in terms of a force displacement curve and $\mathbf{b}$ final necking diameter 
Table 2 parameters) to $10.8 \%$ for optimized $\mathrm{J}-\mathrm{C}$ model with 3 parameters and to $5.8 \%$ for optimized $\mathrm{J}-\mathrm{C}$ model with two parameters. The mesh of the numerical model could be further refined for this kind of analysis, but in any case these results (with a coarser mesh) are fully promising.

It should not come as a surprise that the value of the optimized $A$ parameter is $A=31.2 \mathrm{MPa}$ a lot smaller than the value $A=300.3 \mathrm{MPa}$ of the analytic approach, which is typically considered to be the yield stress of the material. In the optimization approach, the physical meaning of the parameters may be lost. In addition, Fig. $11 \mathrm{~b}$ shows that a good interpolation can be reached also by reducing the number of parameters (e.g. setting $A=0$ ). This is a purely mathematical operation. If the physical meaning of a parameter has to be maintained, the method allows to fix the value of this specific parameter and perform the optimization on the remaining ones.

Figure 12a presents the results of the optimization with the $\mathrm{J}-\mathrm{C}$ model utilising a larger experimental dataset comprising data from all three static tests at $20{ }^{\circ} \mathrm{C}$ (the three numerical curves are practically overlapping as the test conditions are identical). Indicative of the stability of the optimization procedure is the fact that the values of the model parameters $A, B$ and $n$ reported in Fig. 11a (based on a single test) differ very little from those reported in Fig. 12a (based on three tests). A final MAPE $=1.9 \%$ demonstrates the accuracy of the interpolation proposed, considering that the variability of the experimental curves is about $1.5 \%$.

Analogously to the check previously discussed regarding the final necking geometry, a similar verification is applied to the static notched-specimen tests at $20^{\circ} \mathrm{C}$. Using the model parameters extracted from the G1 specimens, the response of the notched G2 specimen has been simulated and the relevant comparison between experimental and predicted numerical curves is shown in Fig. 12b.

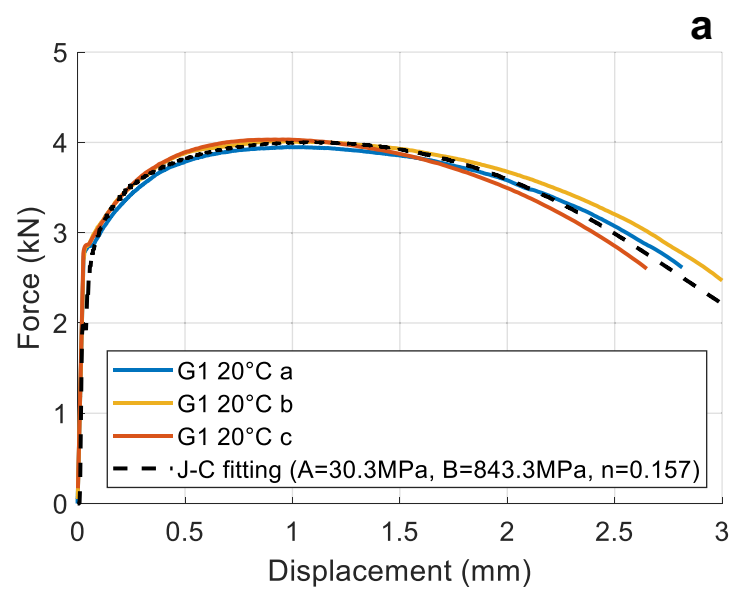

Considering that the material model employed is not triaxiality dependent, it can be concluded that the simulated data interpolate satisfactorily the experimental data with an interpolation error of MAPE $=3.8 \%$. This, once again, proves the capability of the proposed inverse method to correctly work also with complex stress-strain fields. In this context, it is worth mentioning that the same good-quality results (not included here due to space limitations) have been obtained when utilising the notched G2 specimen tests at $20^{\circ} \mathrm{C}$ as experimental dataset for the optimization, and next using the extracted parameters to simulate the tests with G1 geometry for verification.

As already mentioned, another substantial improvement of the inverse methods in the material mechanical characterization lies in the accurate evaluation of the specimen thermal field without considerable increase of computational costs. Figure 13 shows the simulated specimen thermal response in two tests performed on geometry $\mathrm{G} 1$ at $20^{\circ} \mathrm{C}$, statically and dynamically. It can be seen that the two numerical models can efficiently provide an insight of the thermal phenomena that occur in the experimental tests. The simulation results confirm that the static test is almost isothermal because the heat generated in the necking zone is quickly conducted to the rest of the specimen. On the contrary, in the high strainrate test, with conduction phenomena being slower compared to the test dynamics, the temperature of the necking zone increases dramatically up to $200{ }^{\circ} \mathrm{C}$.

Having assessed the potentialities of the approach with the simpler static tests, the inverse method is next applied, utilising appropriately the several tests performed with the cylindrical G1 specimens, in order to obtain all material model parameters. Figure 14 shows the results of this exercise for the $\mathrm{G} 1$ geometry at $20^{\circ} \mathrm{C}$ (three replicates for each test velocity), where additional parameters have been introduced in the FEM models to take account of the strain-rate a

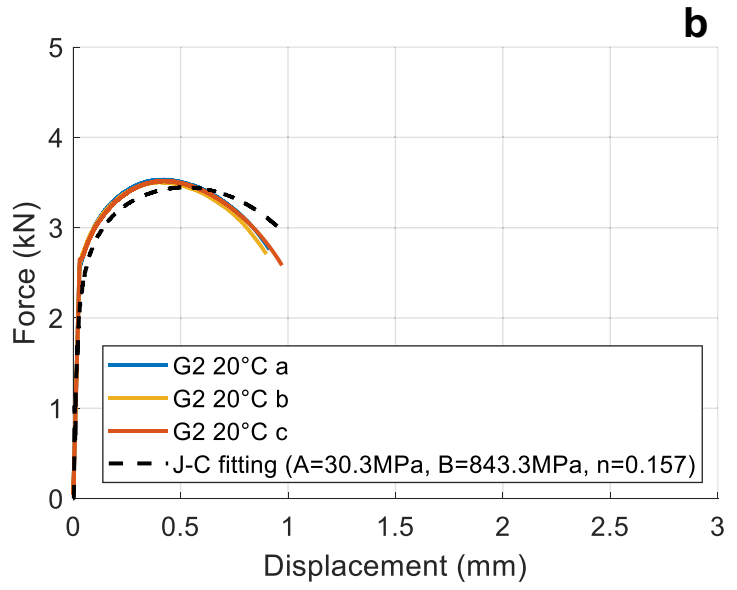

Fig. 12 Comparison of experimental-simulated data for quasi-static test at $20{ }^{\circ} \mathrm{C}$ on a cylindrical (geometry G1) and b notched specimens (geometry G2) 

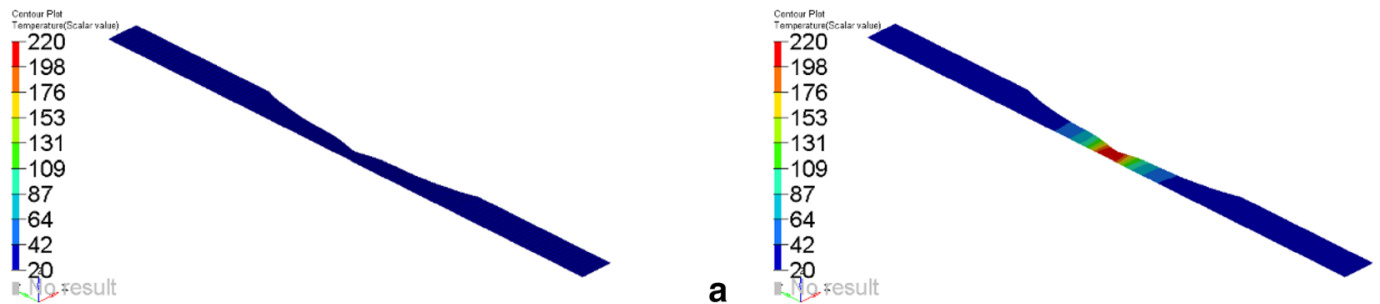

b

Fig. 13 Specimen thermal fields in static and dynamic tests at $20^{\circ} \mathrm{C}$ (geometry G1)

a

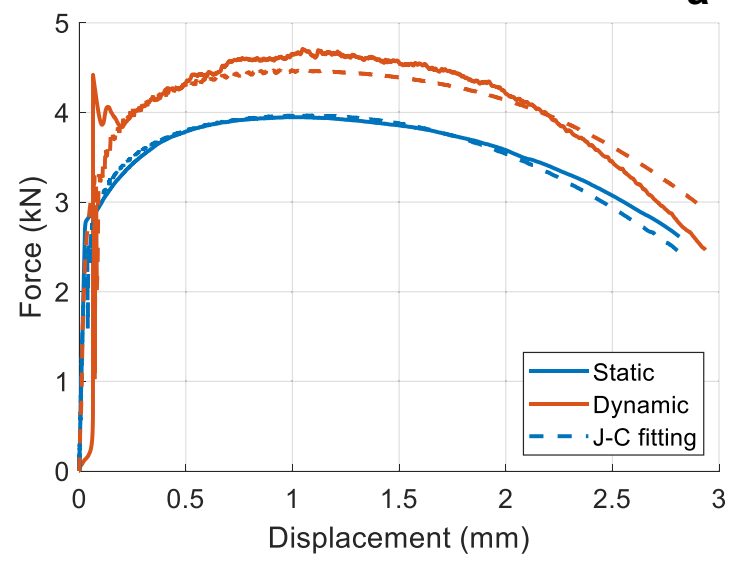

b

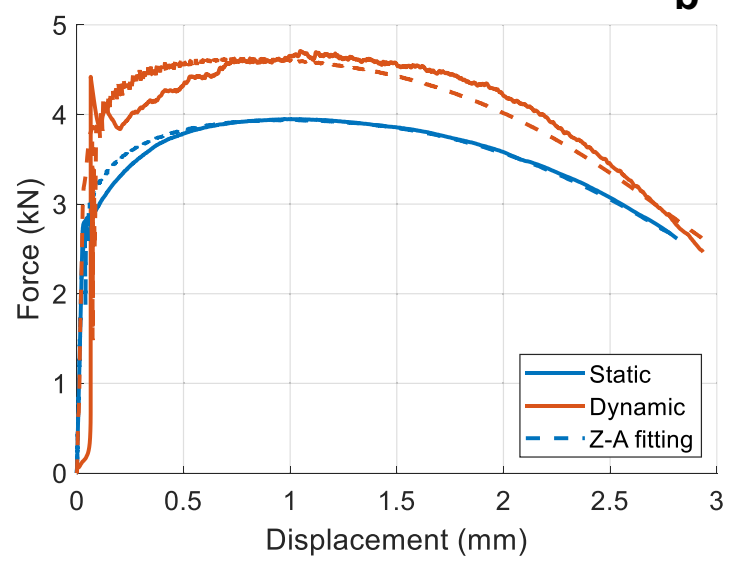

Fig. 14 Comparison of experimental-simulated data for static and dynamic tests (geometry G1) at $20{ }^{\circ} \mathrm{C}$ with a J-C model and b Z-A model

sensitivity ( $c$ parameter for the $\mathrm{J}-\mathrm{C}$ model and constants $B_{0}$, $\beta_{0}$ and $\beta_{1}$ for the Z-A model). In the specific case, also the strain-hardening parameters of the J-C $(A, B$ and $n)$ and Z-A $\left(\mathrm{c}_{0}, K\right.$ and $n$ ) models have been optimized once more, aiming at reducing the interpolation errors in view of the enhanced experimental data set. Of course, an alternative could have been to maintain the strain hardening parameters obtained using only static tests (Fig. 12), and, in this phase, to optimize only the strain-rate sensitivity parameters.

The resulting optimized model parameters are as follows. J-C: $A=29.7 \mathrm{MPa}, B=835.4 \mathrm{MPa}, n=0.155, c=0.028$, and Z-A: $c_{0}=19.3 \mathrm{MPa}, K=619.9 \mathrm{MPa}, n=0.200$, $B_{0}=333.7 \mathrm{MPa}, \beta_{0}=0.013$ and $\beta_{1}=0.0018$. Clearly, both models are able to adequately capture the material strain-rate sensitivity (Fig. 14), as also demonstrated by interpolation errors (MAPE) of $2.7 \%$ and $2.4 \%$, respectively.

Using the model parameters just extracted, Fig. 15 shows the results of verification checks concerning the experimental data of the notched-specimen tests (G2 and G3 geometries) at $20^{\circ} \mathrm{C}$. For the smaller G2 geometry, Fig. 15a, the simulation and experimental curves are very close with an interpolation error MAPE of $4.4 \%$ and $2.5 \%$, respectively, for the J-C and Z-A models. It appears that the Z-A model performs better in the simulation of the tri-axial stress-strain field. However, considering in Fig. 15b the comparison of the simulated curve with the experimental data for the larger G3 specimens, it is evident that the results are less accurate (interpolation error MAPE of $17.6 \%$ and $22.6 \%$, respectively, for the J-C and Z-A models).

This may suggest the presence of possible scale effects [26] on the material properties and work is under way for clarifying this issue. In fact differences exists in the average values of fracture strains for the small G2 and the larger G3 notched specimens, calculated using the final (post mortem) and initial diameter at the minimum section. The values are as reported in Table 3.

Finally, Fig. 16 shows the application of the inverse method to all data concerning the tests on G1 geometry at different velocities and temperatures (three replicates for each test condition) using the $\mathrm{J}-\mathrm{C}$ model (results with the $\mathrm{Z}-\mathrm{A}$ model are of similar quality). In this case all $\mathrm{J}-\mathrm{C}$ parameters $(A, B, n, c, m)$ have been introduced in the FEM models trying to model both thermal and strain-rate sensitivities. Once again, strain-hardening and strain-rate sensitivity parameters have been re-optimized $(A=53.0 \mathrm{MPa}$, $B=781.4 \mathrm{MPa}, n=0.135, c=0.055 m=0.667)$. As seen and previously discussed, the chosen models are able to correctly keep into account the strain-rate sensitivity (blue curves) 

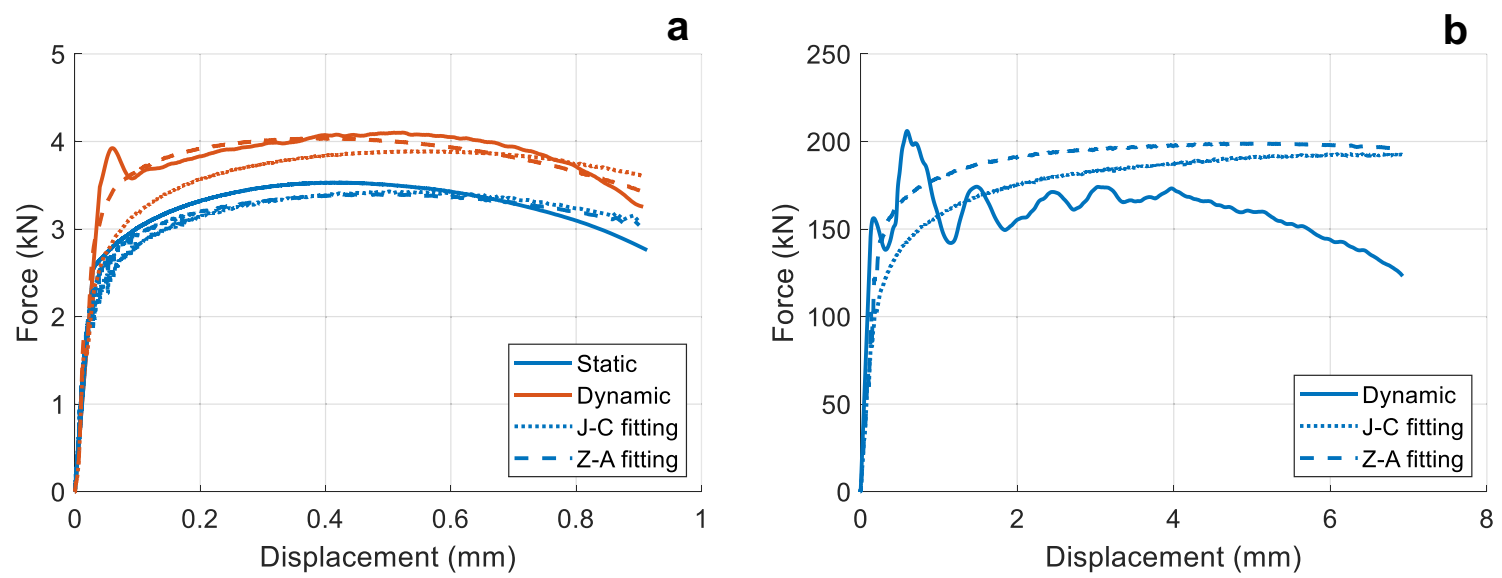

Fig. 15 Comparison of experimental-simulated data for static and dynamic tests at $20^{\circ} \mathrm{C}$ on a small G2 and b large G3 specimens

Table 3 Average values of fracture strains from notched specimens

\begin{tabular}{lllll}
\hline Test type & $\begin{array}{l}\text { Specimen } \\
\text { geometry }\end{array}$ & $\mathrm{T}=20^{\circ} \mathrm{C}$ & $\mathrm{T}=400{ }^{\circ} \mathrm{C}$ & $\mathrm{T}=850{ }^{\circ} \mathrm{C}$ \\
\hline Dynamic test (250/s) & $\mathrm{G} 2$ & 0.860 & 1.030 & 1.630 \\
Dynamic test (200/s) & $\mathrm{G} 3$ & 0.682 & 0.834 & 1.302 \\
\hline
\end{tabular}

but they interpolate poorly data at higher temperatures, as indicated by a mean interpolation error of $\mathrm{MAPE}=13.8 \%$.

\section{Conclusions}

The work presented deals with the problem of identification the parameters of constitutive equations in order to reliably describe the material behaviour. In this direction, the paper has attempted to introduce a new analysis of data, using a rich experimental dataset, which comprises static and dynamic tension tests at room and higher temperatures of two-size smooth and notched cylindrical specimens of a ferritic nuclear steel. The material constitutive models selected and tested are those of $\mathrm{J}-\mathrm{C}$ and $\mathrm{Z}-\mathrm{A}$.

The standard approach of fitting the stress-strain experimental data with the model analytical curves has first been tried and an initial set of model parameter values has been determined. The quality of the fitting, measured by the MAPE between experimental and model predicted stress-strain diagrams, is satisfactory for small strains but poor for larger strains and temperatures. It is also noted as a general comment that both models are not capable of coping adequately with the complex behaviour of the tested material, which seems not to be representable either by a purely multiplicative (J-C) or a purely additive (Z-A) model.

As an alternative to this conventional analytical approach, the paper next examines the adoption of an inverse method, which exploits a FEM model to accurately take into account the specimen stress, strain, and temperature fields. The

a
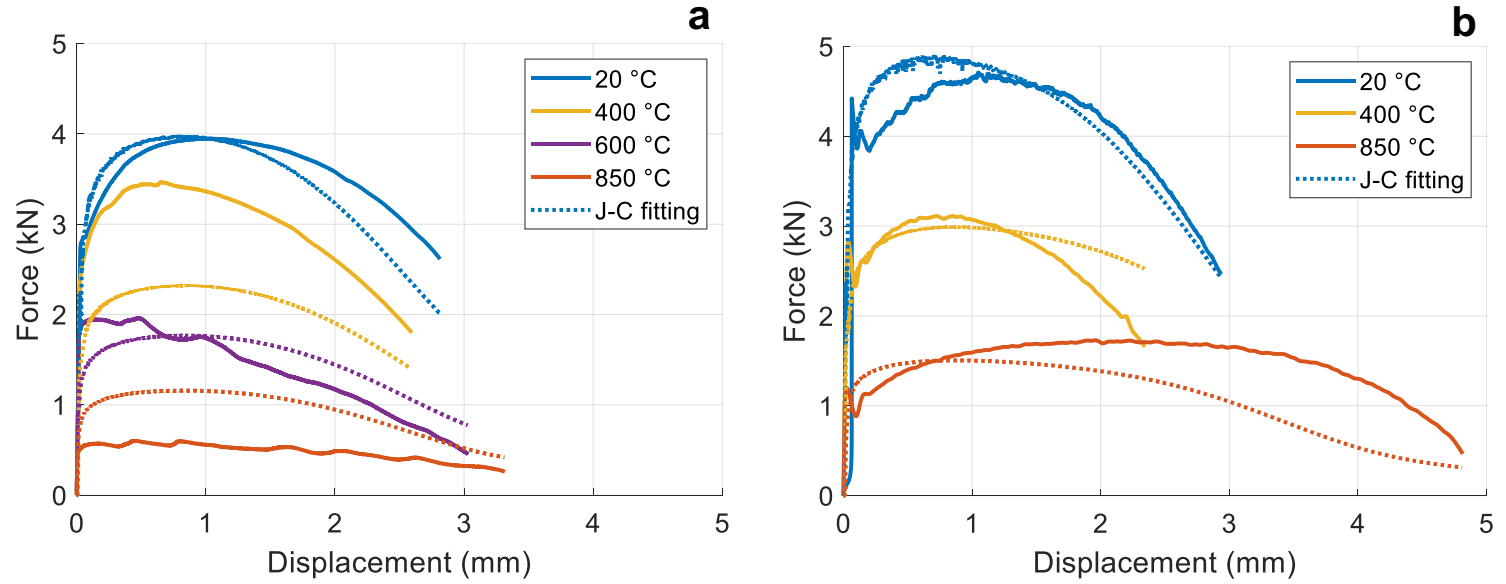

Fig. 16 Comparison experimental-simulated data for $\mathbf{a}$ static and $\mathbf{b}$ dynamic tests (geometry G1) 
whole experimental force-displacement curves, which represent an averaged and more global behaviour of the material specimen, are now being utilised. The material parameters of the two constitutive models ( $\mathrm{J}-\mathrm{C}$ and $\mathrm{Z}-\mathrm{A}$ ) are obtained by using an optimization algorithm that iteratively changes the parameters values aiming to minimize a target function, which is chosen to be the MAPE of the experimental and model predicted force-displacement curves. The approach has been implemented as an automated procedure in MATLAB and uses the LS-DYNA as FEM solver.

An in-depth analysis of the methodology has been provided with simple examples to assess the main advantages of the technique. As shown, the procedure is able to operate on mechanical tests with complex specimen geometries (e.g. notched) and stress conditions (necking in tension testing). Verification exercises have been conducted to assess the accuracy of the predictions through comparisons with experimental data not previously used in the parameter identification phase.

The performance of the approach is generally better than the previously used technique of stress-strain data fitting. Attention is drawn to the fact that, resulting from a mathematical operation, the optimized values of some parameters may not be representative of their physical significance (e.g. yield stress). As explained, this situation can be overcome by fixing the value of the specific parameter(s) and perform the optimization on the rest. Both $\mathrm{J}-\mathrm{C}$ and $\mathrm{Z}-\mathrm{A}$ models manage to reproduce faithfully the response of the specimen (smooth and notched) at lower temperatures under both static and dynamic conditions. Results are not as satisfactory at the higher temperatures, which however may be due to the peculiar behaviour of the material itself, as explained above. The method should be tried with test data from other materials and certainly several of its aspects must be further clarified. As the method has been applied with the main objective to explore its potentialities, it can be concluded that in this respect it has proven efficient and promising.

Open Access This article is distributed under the terms of the Creative Commons Attribution 4.0 International License (http://creativecommons.org/licenses/by/4.0/), which permits unrestricted use, distribution, and reproduction in any medium, provided you give appropriate credit to the original author(s) and the source, provide a link to the Creative Commons license, and indicate if changes were made.

\section{References}

1. Johnson GR, Cook WH (1983) A constitutive model and data for metals subjected to large strains, high strain rates and high temperatures. Proceedings of the 7th International Symposium on Ballistics, The Hague, 1983

2. Zerilli FJ, Armstrong RW (1990) Description of tantalum deformation behaviour by dislocation mechanics based constitutive relations. J Appl Phys 68(4):1580-1591. https:// doi.org/10.1063/1.346636

3. Azodi D, Gruner P (1997) Numerical simulation of a BWR vessel lower head with penetration subjected to a postulated core damage accident. Proc SMiRT 14:443-450

4. Barlat MJ, Knott JF (2007) Simulated coarse-grained heataffected-zone microstructures in DIN 22NiMoCr37 steel. J Nucl Mater 361(1):112-120. https://doi.org/10.1016/j.jnucm at.2006.11.012

5. Standard ASTM A 508/A 508M-05b

6. Rl Krieg et al (2001) Limit strains for severe accident conditions, description of an European research program and first results. Proc SMiRT 16:1226

7. Koc P, Štok B (2004) Computer-aided identification of the yield curve of a sheet metal after onset of necking. Comput Mater Sci 31:155-168. https://doi.org/10.1016/j.commatsci.2004.02.004

8. Ling Y (1996) Uniaxial true stress-strain after necking. Amp J Tech 5:37-48

9. Ghouati O, Gelin JC (1998) Identification of material parameters directly from metal forming processes. J Mater Process Technol 80-81:560-564. https://doi.org/10.1016/S0924 -0136(98)00159-9

10. Meuwissen M, Oomens C, Baaijens F, Petterson R, Janssen J (1998) Determination of the elasto-plastic properties of aluminium using a mixed numerical-experimental procedure. J Mater Process Technol 75(1-3):204-211. https://doi.org/10.1016/ S0924-0136(97)00366-X

11. Kajberg J, Lindkvist G (2004) Characterization of materials subjected to large strains by inverse modelling based on in-plane displacement fields. Int J Solids Struct 41(13):3439-3459. https ://doi.org/10.1016/j.ijsolstr.2004.02.021

12. Endelt B, Nielsen KB (2005) General framework for analytical sensitivity analysis for inverse identification of constitutive parameters. Proceedings COMPLAS, Barcelona, 2005

13. Kleinermann JP (2000) Identification paramétrique et optimisation des procédés de mise a forme par problemes inverses. Dissertation, Université de Liège

14. Flores P (2006) Development of experimental equipment and identification procedures for sheet metal constitutive laws. Dissertation, Université de Liège

15. Dinescu D, Sol H, Hoes K (2002) RTM preform permeability identification by an iterative inverse technique. WIT Trans Built Environ 59:557-566. https://doi.org/10.2495/HPS020551

16. Sol H, De Visscher J, Hua H, Vantomme J, De Wilde WP (1996) La Procédure Résonalyser. La revue des Laboratoires d'essais, Vol. 46

17. Sol H, Hua H, De Visscher J, Vantomme J, De Wilde WP (1997) A mixed numerical/experimental technique for the non-destructive identification of the stiffness properties of fibre reinforced composite materials. NDT\&E Int 30(2):88-91. https://doi. org/10.1016/S0963-8695(96)00049-7

18. Shi Y, Sol H, Hua H (2005) Transverse shear modulus identification by an inverse method using measured flexural resonance frequencies from beams. J Sound Vib 285(1-2):425-442. https ://doi.org/10.1016/j.jsv.2004.03.074

19. Ghouati O, Gelin JC (2001) A finite element-based identification method for complex metallic material behaviour. Comput Mater Sci 21:57-68. https://doi.org/10.1016/S0927-0256(00)00215-9

20. Peroni M (2008) Experimental methods for material characterization at high strain-rate: analytical and numerical improvements. Dissertation, Politecnico di Torino

21. Peroni M, Scapin M, Peroni L (2010) Identification of strainrate and thermal sensitive material model with an inverse method. EPJ Web Conf 6:39004. https://doi.org/10.1051/epjco nf/20100639004 
22. Abertini C, Montagnani M (1974) Testing techniques based on the split Hopkinson bar. Inst Phys Conf 21:22-32

23. Peroni M, Caverzan A, Solomos G (2016) A new apparatus for large scale dynamic tests on materials. Exp Mech 56(5):785796. https://doi.org/10.1007/s11340-015-0123-0

24. Klepaczko JR, Rusinek A, Zaera R (2007) Constitutive relations in 3-D for a wide range of strain rates and temperatures-application to mild steels. Int J Solids Struct 44:5611-5634. https:// doi.org/10.1016/j.ijsolstr.2007.01.015

25. Pierron F, Grédiac M (2012) The virtual field method. SpringerVerlag, New York
26. Solomos G, Albertini C, Labibes K, Pizzinato V, Viaccoz B (2004) Strain rate effects in nuclear steel at room and higher temperatures. Nucl Eng Des 229:139-149. https://doi.org/10.1016/j. nucengdes.2003.10.006

Publisher's Note Springer Nature remains neutral with regard to jurisdictional claims in published maps and institutional affiliations. 